\title{
A PRODUCT VARIETY OF GROUPS WITH DISTRIBUTIVE LATTICE
}

\author{
L. F. HARRIS
}

Abstract. By a variety of A-groups is meant a locally finite variety of groups whose nilpotent groups are abelian. It is shown that if $\mathfrak{U}$ is a variety of $A$-groups and $\mathfrak{B}$ is a locally finite variety whose lattice of subvarieties is distributive and the exponents of $\mathfrak{U}$ and $\mathfrak{B}$ are coprime, then the lattice of subvarieties of the product variety $\mathfrak{U} \mathfrak{B}$ is distributive.

1. Introduction. The lattice of a variety $\mathfrak{B}$ of groups is the lattice of subvarieties of $\mathfrak{B}$ partially ordered by inclusion. It is modular because the lattice of the variety of all groups is dual to the lattice of fully invariant subgroups of the free group of countably infinite rank. For any positive integer $m$ let $\mathfrak{A}_{m}, \mathfrak{B}_{m}$ and $\mathfrak{N}_{m}$ denote respectively the variety of all abelian groups of exponent dividing $m$, the variety of all groups of exponent dividing $m$, and the variety of all groups which are nilpotent of class at most $m$. A variety of A-groups is defined to be a locally finite variety whose nilpotent groups are abelian. G. Higman [7, 54.24] gave the first example of a variety with a nondistributive lattice. R. A. Bryce $[3,6.2 .5]$ showed that for a prime $p$ the product variety $\mathfrak{U}_{p^{2}} \mathfrak{A}_{p^{2}}$ has a nondistributive lattice but that a variety of metabelian groups of bounded exponent in which, for each $p$, the $p$-groups have class at most $p$ has distributive lattice. He also showed that if $m$ is nearly prime to $n$ (i.e. if a prime $p$ divides $m$ then $p^{2}$ does not divide $n$ ) then the lattice of $\mathfrak{A}_{m} \mathfrak{A}_{n}$ is distributive. M. S. Brooks [1] showed that the lattice of $\mathfrak{A}_{3} \mathfrak{A}_{9}$ is not distributive. The main result here generalizes one of John Cossey [4] who showed that the lattice of varieties of $A$-groups is distributive. The exponent of a locally finite variety is defined to be the order of the free group on one generator of the variety.

TheOREM 1. Suppose $\mathfrak{U}$ is a variety of A-groups and $\mathfrak{B}$ is a locally finite variety with distributive lattice and the exponents of $\mathfrak{U}$ and $\mathfrak{B}$ are coprime. Then the lattice of $\mathfrak{U} \mathfrak{B}$ is distributive.

Notation and terminology not here defined are as in Hanna Neumann [7]. In view of Theorem 1 it is worth noting that L. G. Kovács has an

Received by the editors June 4, 1973.

AMS (MOS) subject classifications (1970). Primary 20E10; Secondary 20 F99.

(C) American Mathematical Society 1974 
unpublished example which shows that although the lattice of the meet $\mathfrak{B}_{8} \wedge \mathfrak{N}_{3}$ is distributive, that of $\left(\mathfrak{B}_{8} \wedge \mathfrak{N}_{3}\right) \mathfrak{U}_{3}$ is not.

ACKNOWLeDGement. This work is part of my Ph.D. thesis done under the supervision of Dr. R. M. Bryant and Dr. L. G. Kovács and I thank them both for frequent discussions and constructive criticism. I also thank Dr. John Cossey who suggested the problem discussed here, and the Australian National University for a Research Scholarship which enabled me to carry out this work.

2. A theorem on skeletons. By a section of a group is meant a factor group of a subgroup of it. If $\mathscr{G}$ is a class of groups then $\mathrm{s} \mathscr{G}$ and $\mathrm{Q} \mathscr{G}$ denote the classes of all groups isomorphic to, respectively, subgroups and factor groups of groups in $\mathscr{G}$. A class $\mathscr{G}$ of groups is said to be section closed if $\mathrm{Q} \mathscr{G} \subseteq \mathscr{G}$ and $\mathrm{s} \mathscr{G} \subseteq \mathscr{G}$. It is well known and easy to see that if $\mathscr{G}$ is a class of groups then QS $\mathscr{G}$ is section closed. The skeleton $\mathscr{S}(\mathfrak{B})$ of a variety $\mathfrak{B}$ is defined (in Bryant and Kovács [2]) to be the intersection of the section closed classes of groups generating $\mathfrak{B}$. A monolithic group is defined to be a finite group with a unique minimal normal subgroup, called the monolith. To prove Theorem 1 we need the following result.

THEOREM 2. Suppose $p$ is a prime and $\mathfrak{Y}$ is a locally finite variety containing a variety $\mathfrak{X}$ of $p^{\prime}$-exponent such that for some positive integer $\alpha, \mathfrak{Y}$ is contained in $\mathfrak{U}_{p a} \mathfrak{X}$,

$$
\mathfrak{X} \subseteq \mathfrak{Y} \subseteq \mathfrak{A}_{p^{\alpha}} \mathfrak{X},
$$

and $\mathfrak{Y}$ is generated by monolithic groups not in $\mathfrak{X}$. Then

$$
\mathscr{S}(\mathfrak{Y})=\mathrm{QS}\{G \mid G \in \mathfrak{Y}, G \notin \mathfrak{X} \text { and } G \text { is monolithic }\},
$$

and $\mathscr{S}(\mathfrak{Y})$ generates $\mathfrak{Y}$.

Proof. Let $G$ be a monolithic group in $\mathfrak{Y}$ but not in $\mathfrak{X}$, let $\sigma G$ be the monolith of $G, \sigma^{*} G$ be the centralizer of $\sigma G$ in $G, Z(G)$ be the center of $G, X=\mathfrak{X}(G)$ be the $\mathfrak{X}$-verbal subgroup of $G$, and $G^{\prime}$ be the derived group of $G$. We write $H \triangleleft G$ if $H$ is a normal subgroup of $G$.

Notice $X$ is the Sylow $p$-subgroup of $\sigma^{*} G$; we show they are equal. If $\sigma^{*} G$ is not abelian then

$$
\sigma G \leqq\left(\sigma^{*} G\right)^{\prime} \cap Z\left(\sigma^{*} G\right) \cap X=1
$$

by [6, IV2.2], which is a contradiction. Thus $\sigma^{*} G$ is abelian and, since $G$ is monolithic, $\sigma^{*} G$ is of prime power order. Because

we have $X=\sigma^{*} G$.

$$
\sigma G \leqq X \leqq \sigma^{*} G,
$$


Let $\mathscr{H}$ be a section closed class of groups generating $\mathfrak{Y}$. To prove the theorem it suffices to show $G \in \mathscr{H}$. We shall use some properties of the minimal representation defined in [7, p. $163 \mathrm{ff}]$. Let

$$
G \cong H / K, \quad H \leqq H_{1} \times \cdots \times H_{r}, H_{i} \in \mathscr{H} \text { for } i=1, \cdots, r
$$

be a minimal representation of $G$ on $\mathscr{H}$. Then each $H_{i}$ is monolithic and $\sigma H_{i} \cong \sigma G$ so $\sigma H_{i}$ is a $p$-group. By the last paragraph $\sigma^{*} H_{i}=\mathfrak{X}\left(H_{i}\right)$. By the Schur-Zassenhaus theorem there is a complement, $K_{i}$ say, for $\sigma^{*} H_{i}$ in $H_{i}$. Since $H_{i}$ is monolithic, $\sigma^{*} H_{i}$ is an indecomposable $K_{i}$-group so by [5, 5.2.2], $\sigma^{*} H_{i}$ is a homocyclic $p$-group. For some $j$ the exponent of $\sigma^{*} H_{j}$ is greater than or equal to the exponent of $\sigma^{*} G$. Let $n$ be the exponent of $\sigma^{*} G$. It follows as in Lemma 3 of Cossey [4] that $G \cong H_{j} /\left(\sigma^{*} H_{j}\right)^{n}$, and $G \in \mathscr{H}$, proving the theorem.

3. Proof of Theorem 1. Let $\mathfrak{U}_{1}, \mathfrak{U}_{2} \leqq \mathfrak{U} \mathfrak{B}$. We first show

$$
\left(\mathfrak{B} \wedge \mathfrak{U}_{1}\right) \vee\left(\mathfrak{B} \wedge \mathfrak{U}_{2}\right)=\mathfrak{B} \wedge\left(\mathfrak{U}_{1} \vee \mathfrak{U}_{2}\right) .
$$

Since $\left(\mathfrak{B} \wedge \mathfrak{U}_{1}\right) \subseteq \mathfrak{B} \wedge\left(\mathfrak{U}_{1} \vee \mathfrak{U}_{2}\right)$ it suffices to prove that if $F$ is a finite free group of $\mathfrak{B} \wedge\left(\mathfrak{U}_{1} \vee \mathfrak{U}_{2}\right)$ then $F \in\left(\mathfrak{B} \wedge \mathfrak{U}_{1}\right) \vee\left(\mathfrak{B} \wedge \mathfrak{U}_{2}\right)$. Let $\mathfrak{U}_{1} \cup \mathfrak{U}_{2}$ denote the set theoretic union of $\mathfrak{U}_{1}$ and $\mathfrak{U}_{2}$. Let

$$
F \cong H / K, \quad H \leqq H_{1} \times \cdots \times H_{r}, H_{i} \in\left(\mathfrak{U}_{1} \cup \mathfrak{U}_{2}\right) \text { for } i=1, \cdots, r
$$

be a minimal representation of $F$ on $\mathfrak{U}_{1} \cup \mathfrak{U}_{2}$. Because $F \in \mathfrak{B}, \sigma H_{i}$ has exponent dividing that of $\mathfrak{B}$. Since the exponents of $\mathfrak{U}$ and $\mathfrak{B}$ are relatively prime it follows that $H_{i} \in \mathfrak{B}$ for all $i$. As $H_{i} \in \mathfrak{U}_{1} \cup \mathfrak{U}_{2}$ we have $H_{i} \in$ $\left(\mathfrak{B} \cap \mathfrak{U}_{1}\right) \cup\left(\mathfrak{B} \cap \mathfrak{U}_{2}\right)$. It follows that $F \in\left(\mathfrak{B} \wedge \mathfrak{U}_{1}\right) \vee\left(\mathfrak{B} \wedge \mathfrak{U}_{2}\right)$, proving $\left(^{*}\right)$.

We need a lemma.

Lemma. If $G$ is a monolithic group in $\mathfrak{U}_{1} \vee \mathfrak{U}_{2}$ but not in $\mathfrak{B}$ then $G \in$ $\mathfrak{U}_{1} \cup \mathfrak{H}_{2}$.

Proof. If $\sigma G$ is not abelian then by taking a minimal representation of $G$ on $\mathfrak{U}_{1} \cup \mathfrak{H}_{2}$ and arguing as in $[7,53.31]$ the result follows. Thus we may assume $\sigma G$ is an abelian $p$-group for some prime $p$. Let

$$
G \cong H / K, \quad H \leqq H_{1} \times \cdots \times H_{r}, H_{i} \in\left(\mathfrak{U}_{1} \cup \mathfrak{U}_{2}\right) \text { for } i=1, \cdots, r
$$

be a minimal representation of $G$ on $\mathfrak{U}_{1} \cup \mathfrak{U}_{2}$. Let $V_{i}=\left(\sigma^{*} H_{i}\right) \cap \mathfrak{B}\left(H_{i}\right)$ and observe that the Sylow $p$-subgroups of $H_{i}$ are in $V_{i}$ and $\sigma H_{i} \leqq$ $Z\left(V_{i}\right)$. As $V_{i}$ is an $A$-group, $Z\left(V_{i}\right) \cap V_{i}^{\prime}=1$. Since $H_{i}$ is monolithic, $V_{i}^{\prime}=1$. Thus $V_{i}$ is abelian and must be a $p$-group.

Let $\mathfrak{Y}$ be the variety generated by $H_{1}, \cdots, H_{r}$ and $\mathfrak{X}$ be the variety generated by $H_{1} / V_{1}, \cdots, H_{r} / V_{r}$. Then by Theorem 2 ,

$$
\mathscr{S}(\mathfrak{Y})=\operatorname{QS}\{H \mid H \in \mathfrak{Y}, H \notin \mathfrak{X} \text { and } H \text { is monolithic }\} .
$$


It follows that

$$
G \in \mathscr{S}(\mathfrak{Y}) \subseteq \operatorname{QS}\left\{H_{1}, \cdots, H_{r}\right\} \subseteq \mathfrak{U}_{1} \cup \mathfrak{U}_{2}
$$

proving the lemma.

To prove Theorem 1 it suffices to show that if $\mathfrak{W} \subseteq \mathfrak{U} \mathfrak{B}$ then

$$
\mathfrak{W} \wedge\left(\mathfrak{U}_{1} \vee \mathfrak{U}_{2}\right)=\left(\mathfrak{W} \wedge \mathfrak{U}_{1}\right) \vee\left(\mathfrak{W} \wedge \mathfrak{U}_{2}\right) \text {. }
$$

Since $\mathfrak{W} \wedge\left(\mathfrak{U}_{1} \vee \mathfrak{U}_{2}\right) \supseteq \mathfrak{W} \wedge \mathfrak{U}_{1}$ it suffices to show that if $G$ is a monolithic group in $\mathfrak{W} \wedge\left(\mathfrak{U}_{1} \vee \mathfrak{U}_{2}\right)$ then $G$ is in $\left(\mathfrak{W} \wedge \mathfrak{U}_{1}\right) \vee\left(\mathfrak{W} \wedge \mathfrak{U}_{2}\right)$.

Suppose first that $G \notin \mathfrak{B}$. Then by the lemma $G \in \mathfrak{U}_{1} \cup \mathfrak{U}_{2}$. As $G \in \mathfrak{W}$,

$$
\begin{aligned}
G \in \mathfrak{W} \cap\left(\mathfrak{U}_{1} \cup \mathfrak{U}_{2}\right) & =\left(\mathfrak{W} \cap \mathfrak{U}_{1}\right) \cup\left(\mathfrak{W} \cap \mathfrak{U}_{2}\right) \\
& \subseteq\left(\mathfrak{W} \wedge \mathfrak{U}_{1}\right) \vee\left(\mathfrak{W} \wedge \mathfrak{U}_{2}\right) .
\end{aligned}
$$

Suppose $G \in \mathfrak{B}$. Using the fact that $\mathfrak{B}$ has distributive lattice and applying (*) twice, we have

$$
\begin{aligned}
\mathfrak{B} \wedge \mathfrak{W} \wedge\left(\mathfrak{U}_{1} \vee \mathfrak{U}_{2}\right) & =\mathfrak{B} \wedge \mathfrak{W} \wedge\left[\mathfrak{B} \wedge\left(\mathfrak{U}_{1} \vee \mathfrak{U}_{2}\right)\right] \\
& =(\mathfrak{B} \wedge \mathfrak{W}) \wedge\left[\left(\mathfrak{B} \wedge \mathfrak{U}_{1}\right) \vee\left(\mathfrak{B} \wedge \mathfrak{U}_{2}\right)\right] \\
& =\left[(\mathfrak{B} \wedge \mathfrak{W}) \wedge\left(\mathfrak{B} \wedge \mathfrak{U}_{1}\right)\right] \vee\left[(\mathfrak{B} \wedge \mathfrak{W}) \wedge\left(\mathfrak{B} \wedge \mathfrak{U}_{2}\right)\right] \\
& =\left(\mathfrak{B} \wedge \mathfrak{W} \wedge \mathfrak{U}_{1}\right) \vee\left(\mathfrak{B} \wedge \mathfrak{W} \wedge \mathfrak{U}_{2}\right) \\
& =\mathfrak{B} \wedge\left[\left(\mathfrak{W} \wedge \mathfrak{U}_{1}\right) \vee\left(\mathfrak{W} \wedge \mathfrak{U}_{2}\right)\right] .
\end{aligned}
$$

This completes the proof of the theorem.

\section{REFERENCES}

1. M. S. Brooks, On lattices of varieties of metabelian groups, J. Austral. Math. Soc. 12 (1971), 161-166. MR 45 \#3526.

2. R. M. Bryant and L. G. Kovács, The skeleton of a variety of groups, Bull. Austral. Math. Soc. 6 (1972), 357-378.

3. R. A. Bryce, Metabelian groups and varieties, Philos. Trans. Roy. Soc. London Ser. A 266 (1970), 281-355. MR 42 \#349.

4. John Cossey, Critical groups and the lattice of varieties, Proc. Amer. Math. Soc. 20 (1969), 217-221. MR 38 \#1147.

5. Daniel Gorenstein, Finite groups, Harper and Row, New York, 1968. MR 38 \#229.

6. B. Huppert, Endliche Gruppen. I, Die Grundlehren der math. Wissenschaften, Band 134, Springer-Verlag, Berlin and New York, 1967. MR 37 \#302.

7. Hanna Neumann, Varieties of groups, Ergebnisse der Math. und ihrer Grenzgebiete, Band 37, Springer-Verlag, New York, 1967. MR 35 \#6734.

Department of Mathematics, Institute of Advanced Studies, Australian National University, Canberra, ACT, Australia

Current address: 8085 King George Highway, Surrey, British Columbia, Canada 